\title{
Conocimientos sobre bioseguridad en estudiantes de medicina de una universidad privada de Chiclayo, Perú, 2018
}

Alejandro Cabrera-Abarca 1,a, Christy Maribel Leyva-Gonzales ${ }^{1, a}$, Percy Fabrizio

Pérez-Pérez 1,a, Elmer López-López 2,b, Antero Enrique Yacarini-Martínez 1,c

\begin{abstract}
RESUMEN
Objetivo. Describir el conocimiento sobre medidas de bioseguridad para el manejo de residuos biocontaminados en estudiantes de primer año de Medicina Humana una universidad de Chiclayo, Perú, durante octubre de 2018. Material y métodos. Estudio descriptivo transversal, para lo cual se aplicó un cuestionario estructurado basado en el Protocolo de Bioseguridad de los Laboratorios tipo I. Resultados. Se estudió 185 estudiantes. La población se caracterizó el predominio de las mujeres (78,5\%). El 30,2\% y el $82,4 \%$ de estudiantes desconocían la información básica con respecto a los pictogramas de seguridad y la forma correcta de eliminación de residuos, respectivamente. Mientras que el 99,4 \% y 95,4 \% conocían sobre la indumentaria correcta y el lavado de manos. Los 99,0 \% de estudiantes de Morfofisiología II respondieron correctamente el ítem sobre lavado de manos. Conclusiones. Una proporción importante de estudiantes de Medicina de la USAT desconoce los pictogramas de seguridad y la eliminación de residuos sólidos, pero conocen la indumentaria y lavado de manos correctos.
\end{abstract}

Palabras Clave: Bioseguridad, Estudiantes de Medicina, Conocimiento (Fuente: DeCS-BIREME).

\section{Knowledge about biosafety in medical students of a private university in Chiclayo, Peru, 2018}

\begin{abstract}
Objetive. Describe the knowledge about biosecurity measures for the management of biocontaminated waste in first year students of Human Medicine at a university in Chiclayo, Peru, during October 2018. Material and methods. Descriptive cross-sectional study, for which a structured questionnaire was applied based on the Laboratory Biosecurity Protocol type I. Results. 185 students were studied. The population was characterized by the predominance of women (78.5\%). $30.2 \%$ and $82.4 \%$ of students were unaware of the basic information regarding safety pictograms and the correct form of waste disposal, respectively. While $99.4 \%$ and $95.4 \%$ knew about the correct clothing and handwashing. 99.0\% of Morphophysiology II students correctly answered the item on handwashing. Conclusions. A significant proportion of USAT Medical students are unaware of the safety pictograms and solid waste disposal, but they know the correct clothing and hand washing.
\end{abstract}

Keywords: Biosafety, Medical Students, Knowledge (Source: MeSH-NLM).

\footnotetext{
${ }_{1}^{1}$ Facultad de Medicina, Universidad Católica Santo Toribio de Mogrovejo, Chiclayo, Perú.

2 Facultad de Medicina, Universidad Señor de Sipán, Chiclayo, Perú.

a Estudiante de Medicina Humana.

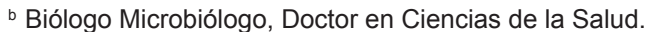

${ }^{\mathrm{b}}$ Biólogo Microbiólogo, Maestría en Ciencias con mención en Microbiología.
} 


\section{INTRODUCCIÓN}

La bioseguridad constituye medidas destinadas a la prevención de cualquier accidente o riesgo laboral que involucre el uso de material biológico, por definición como los principios, técnicas y prácticas aplicadas a fin de evitar la exposición no intencional a agentes de riesgo biológico y toxinas, o su liberación accidental ${ }^{(1,2)}$. La importancia de la bioseguridad está en el manejo de microorganismos patógenos en los laboratorios de enseñanza, además de minimizar el riesgo para los estudiantes y la comunidad en general ${ }^{(3)}$. A nivel mundial, el $20 \%$ de los residuos que generan las actividades de atención sanitaria, son considerados material peligroso de tipo infeccioso, tóxico o radioactivo ${ }^{(4)}$. Aproximadamente cada año se administran en el mundo 16 billones de inyecciones, pero no todas las agujas y jeringas se descartan correctamente ${ }^{(5)}$. La exposición ocupacional a sangre y fluidos corporales es una preocupación seria para los trabajadores de la salud, y presenta un riesgo importante de transmisión de infecciones por el Virus de la Inmunodeficiencia Humana $(\mathrm{VIH})$ y los virus de la hepatitis ${ }^{(6)}$. Los proveedores de atención médica (PS) están en riesgo de exposición ocupacional a la infección por el $\mathrm{VIH}{ }^{(7)}$. La Organización Mundial de la Salud (OMS) estima que la carga global de infección por el $\mathrm{VIH}$ debido a exposición ocupacional es del 2,5\% entre los PS ${ }^{(8)}$. Se estima que el $90 \%$ de estas exposiciones ocupacionales ocurren en países en desarrollo debido a la falta general de conciencia, educación y capacitación estructurada sobre prevención y medidas de exposición a la infección por el virus ${ }^{(9)}$.

El buen conocimiento de los educadores sobre las prácticas estándares de bioseguridad contribuye a garantizar laboratorios de enseñanza más seguros ${ }^{(3)}$. Por ello, el correcto manejo y segregación de los residuos biocontaminados es materia de suma relevancia en el ámbito médico y en la formación de los futuros profesionales de la salud, como los médicos, ya que de esta manera se procura no poner en riesgo a la población por un inadecuado empleo y descarte de muestras biológicas.

El objetivo del presente estudio fue determinar el conocimiento sobre medidas de bioseguridad en el manejo de residuos biocontaminados, en estudiantes de Medicina del primer año de la Universidad Católica Santo Toribio de Mogrovejo (USAT).

\section{MATERIAL Y MÉTODOS}

\section{Tipo y diseño de investigación}

Estudio descriptivo de corte transversal.

\section{Población y muestra}

El estudio fue censal con 197 participantes estudiantes del primer año de la Escuela de Medicina Humana que cursaron las asignaturas con uso práctico de laboratorio (Bioquímica,
Morfofisiología II, Microbiología y Parasitología, y Fisiopatología II). Se incluyeron a los estudiantes que aceptaron participar mediante firma de consentimiento informado y se excluyeron a aquellos que se retiraron durante el proceso de investigación.

\section{Técnicas e instrumentos de recolección de dato}

Para describir los conocimientos se utilizó un cuestionario de 10 preguntas de cuatro alternativas, elaborado por los investigadores en base al Protocolo de Bioseguridad de los Laboratorios tipo I y revisado por dos microbiólogos. Cada pregunta se calificó por separado y se describieron las frecuencias de preguntas correctamente respondidas. Se utilizó la media como medida de tendencia central y medidas de dispersión como la desviación estándar.

\section{Consideraciones éticas.}

El estudio fue aprobado por el Comité de Ética en Investigación de la Facultad de Medicina de la Universidad Católica Santo Toribio de Mogrovejo.

\section{Análisis de datos}

Se analizaron los datos utilizando estadística descriptiva usando el software Microsoft Excel 2013.

\section{RESULTADOS}

Se estudiaron 185 estudiantes. El promedio de edad de la población encuestada fue de 19,05 años, con una desviación estándar de $\pm 1,58$ años. El número de mujeres encuestadas fue de $121(64,8 \%)$ y el de hombres encuestados fue de $64(35,2 \%)$.

Se observó un elevado índice de estudiantes que conocen la indumentaria $(99,4 \%)$ y el lavado de manos $(95,4$ $\%$ ), siendo las preguntas con mayor porcentaje de acierto; mientras que desconocen información básica con respecto a los pictogramas de seguridad $(30,2 \%)$ y la forma correcta de eliminación de residuos $(82,1 \%)$, que constituyen las dos preguntas con menor porcentaje de aciertos del cuestionario. Ver figura 1.

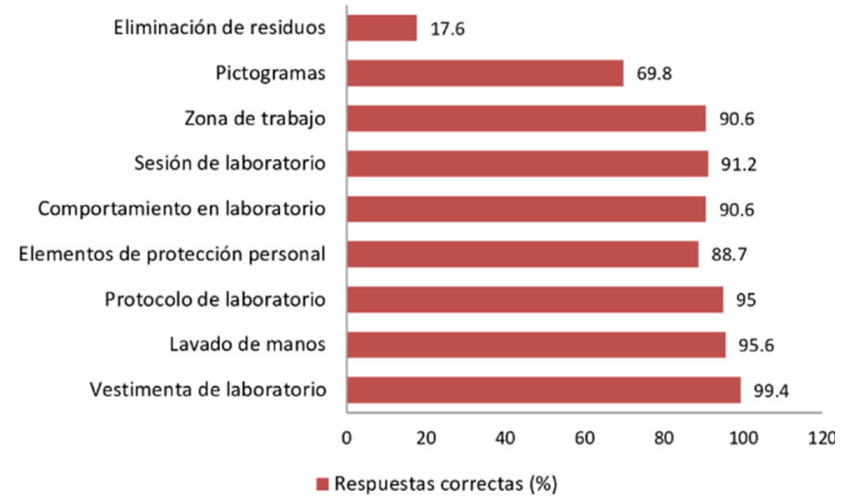

Figura 1. Porcentaje de preguntas respondidas correctamente por estudiantes de medicina de una universidad de Lambayeque - Perú. 2018. 
Por curso se observó que todos los alumnos pertenecientes a las asignaturas de Bioquímica, Microbiología y Parasitología, y Fisiopatología II acertaron en el primer ítem sobre vestimenta de laboratorio. Por otra parte, todos los estudiantes de la asignatura de Microbiología y Parasitología también respondieron correctamente los ítems sobre lavado de manos y protocolo de bioseguridad, siendo esta la única materia en donde todos los alumnos respondieron correctamente más de un ítem. Además, se observó una lata proporción de estudiantes de la asignatura de Morfofisiología II (99\%) respondió correctamente el ítem sobre lavado de manos. Ver tabla 1.

Tabla 1. Frecuencia de respuestas correctas sobre medidas de bioseguridad en estudiantes de primer año de medicina de una universidad privada de Chiclayo, Perú. 2018.

\begin{tabular}{|c|c|c|c|}
\hline Asignatura & $\mathbf{N}$ & $\%$ & Aspecto de bioseguridad \\
\hline Bioquímica & 70 & 100 & Vestimenta de laboratorio \\
\hline Fisiopatología II & 29 & 100 & Vestimenta de laboratorio \\
\hline $\begin{array}{l}\text { Microbiología y parasi- } \\
\text { tología }\end{array}$ & 18 & 100 & $\begin{array}{l}\text { Vestimenta de laboratorio, la- } \\
\text { vado de manos, protocolo de } \\
\text { laboratorio }\end{array}$ \\
\hline Morfofisiología II & 67 & 99 & Lavado de manos \\
\hline
\end{tabular}

La pregunta sobre eliminación de residuos obtuvo la menor cantidad de respuestas correctas en todas las asignaturas,-siendo Fisiopatología II (100\%) y Microbiología y Parasitología $(94,4 \%)$ los dos cursos con menor cantidad de estas. Ver tabla 2.

Tabla 2. Frecuencia de respuestas incorrectas sobre medidas de bioseguridad en estudiantes de primer año de medicina de una universidad privada de Chiclayo, Perú. 2018

\begin{tabular}{lccc}
\hline Asignatura & N & $\%$ & Aspecto de bioseguridad \\
\hline Bioquímica & 45 & 64,3 & Eliminación de residuos \\
$\begin{array}{l}\text { Fisiopatología II } \\
\begin{array}{l}\text { Microbiología y y } \\
\text { parasitología }\end{array}\end{array}$ & 17 & 94,4 & Eliminación de residuos \\
\begin{tabular}{l} 
Morfofisiología II \\
\hline \hline
\end{tabular} & 56 & 82,4 & Eliminación de residuos \\
\hline
\end{tabular}

\section{DISCUSIÓN}

Dentro de las carreras biomédicas, el conocimiento sobre medidas de bioseguridad es importante debido a que evitan la propagación y el contagio accidental de agentes patógenos hacia el personal de salud y a la población en general ${ }^{(10)}$. Así, dichos conocimientos deben ser adquiridos durante el proceso de formación de los estudiantes y ser evaluados constantemente para que puedan integrarse a su comportamiento cotidiano y ponerse en práctica durante su labor clínica ${ }^{(11,12)}$.

A nivel global, en nuestro estudio se observó un índice alto de estudiantes que posee un alto conocimiento sobre el uso de indumentaria $(99,4 \%)$ y el lavado de manos $(95,4 \%)$, siendo las preguntas con mayor porcentaje de acierto, mientras que los ítems con menor cantidad de aciertos son acerca de los pictogramas de seguridad $(69,8 \%)$ y la forma correcta de eliminación de residuos $(17,6 \%)$, dichos resultados se corroboran con otro realizado por Díaz en Perú en el 2012, en un estudio descriptivo transversal en población universitaria de la carrera de Enfermería de la Universidad Nacional de la Amazonía Peruana, donde halló que el $92,6 \%$ de la población encuestada respondió correctamente los ítems sobre el lavado de manos y el uso de guantes estériles ${ }^{(13)}$. En cambio en otro estudio llevado a cabo en estudiantes casi el $68,6 \%$ de estudiantes desconocían la secuencia de lavarse las manos ${ }^{(14)}$.

Chero en Perú en el 2016, en un estudio descriptivo transversal en población universitaria halló que el $83,3 \%$ posee un nivel de conocimiento alto en el uso de guantes, mientras que en relación a la eliminación de residuos contaminantes es bajo en un $43,3 \%$. Dicho resultado es alarmante, ya que puede estar relacionado a la poca habituación que poseen los estudiantes por la utilización de los objetos punzantes y cortantes durante sus sesiones prácticas ${ }^{(15)}$. La transmisión de patógenos nosocomiales de paciente a paciente se ha relacionado con la colonización transitoria de los trabajadores de la salud, y los estudios han sugerido que la contaminación de la ropa de los trabajadores de la salud, incluidas las batas blancas, puede ser un vector para esta transmisión ${ }^{(16-18)}$.

Estos resultados muestran los conocimientos que poseen los alumnos de la población estudiada respecto a los protocolos de bioseguridad en laboratorio, mas no evidencia una adecuada correlación en la práctica por la mayoría de la población estudiantil. Nuestra recomendación es brindar un mayor énfasis sobre el reconocimiento de la señalización de laboratorio y diseñar un plan práctico sobre una correcta segregación de residuos durante la capacitación de los alumnos, para así poder contribuir a una óptima aplicación del protocolo de bioseguridad de los laboratorios en su totalidad.

En este estudio no se evaluó la parte actitudinal con respecto al cumplimiento de los protocolos de bioseguridad, los resultados se basan únicamente en las encuestas respondidas por los estudiantes. No obstante, la falta de correlación entre los aspectos teórico y práctico presentada se ve respaldada ya que durante el proceso de evaluación se observó que los alumnos llevaban puesto el guardapolvo de bioseguridad en los corredores y espacios públicos. Una mejor comprensión de las percepciones, actitudes y conocimiento de los estudiantes de medicina sobre la bioseguridad podría facilitar una educación más efectiva de los futuros médicos sobre estos temas importantes. 
Las limitaciones del estudio son la reducida cantidad de participantes que se incluyeron en el estudio y que solo se censó una universidad. Las preguntas debieron haber pasado antes por un estudio piloto el cual no hubiese podido mejorar el enfoque de las preguntas en el estudio. Se debió ampliar el estudio a todas las universidades de la región, incluir también a los docentes ya que son estos los que imparten los conocimientos en las clases.

Esta investigación nos da a entender que puede que en la malla curricular no se contempla una asignatura específica teórico - práctica con respecto a la capacitación de medidas de bioseguridad, además de las pocas iniciativas de los hospitales donde se enfatice y obligue al estudiante a la aplicabilidad de los protocolos de bioseguridad.

En conclusión, el conocimiento sobre bioseguridad corresponde a un aspecto académico y formativo en el estudiante de Medicina de suma importancia por su aplicación en su futura labor asistencial, la cual posee participación docente a lo largo de los años de pregrado. En el estudio realizado, se observa que el conocimiento de bioseguridad por parte de estudiantes de pregrado de las carreras biomédicas presenta un alto desarrollo en materia de equipo de protección personal y protocolos de bioseguridad, mas no se evidencia lo mismo en aquellos aspectos que no son puestos en práctica constantemente por la naturaleza de las experiencias, como reconocimiento de señalización y segregación de residuos biológicos.

Fuentes de financiamiento. Financiado por los autores.

Conflictos de intereses. Los autores señalan no tener conflictos de interés.

\section{REFERENCIAS BIBLIOGRÁFICAS}

1. Ayón-Haro ER, Villanelo-Ninapaytan MS, Bedoya-Arboleda L, González-Chávez R, Pardo-Aldave K, Picasso-Pozo MÁ, et al. Conocimientos y actitudes sobre bioseguridad en estudiantes de odontología de una universidad peruana. Kiru. 2014;11(1):39-45.

2. Sutcliffe C, Quinn $\mathrm{CH}$, Shannon C, Glover A, Dunn AM. Exploring the attitudes to and uptake of biosecurity practices for invasive non-native species: views amongst stakeholder organisations working in UK natural environments. Biol Invasions. 2018; 20(2):399-411.

3. Cruz CP, Cruz JP, Abu Bakr SA, Thazha SK. Biosafety knowledge and perceptions of clinical laboratory science educators in a Saudi University. Internactonal J Heal Sci Res. 2015; 5(6):196-204.

4. Pépin J, Chakra CNA, Pépin E, Nault V, Valiquette L. Evolution of the global burden of viral infections from unsafe medical injections, 2000-2010. PLoS One. 2014; 9(6): e99677

5. WHO WHO. Management of waste from injection activities at district level Guidelines for District Health Managers. 2006;1-20.

6. Reda AA, Fisseha S, Mengistie B, Vandeweerd J-M. Standard precautions: occupational exposure and behavior of health care workers in Ethiopia. PLoS One. 2010; 5(12):e14420.

7. Gupta A, Anand S, Sastry J, Krisagar A, Basavaraj A, Bhat SM, et al. High risk for occupational exposure to HIV and utilization of post-exposure prophylaxis in a teaching hospital in Pune, India. BMC Infect Dis. 2008; 8:142.
8. Guilbert JJ. The world health report 2002 - Reducing risks, promoting healthy life [2]. Vol. 16, Education for Health. World Health Organization; 2003. p. 230.

9. Sagoe-Moses C, Pearson RD, Perry J, Jagger J. Risk to health care workers in developing countries. Vol. 345, New England Journal of Medicine. 2001. p. 538-41.

10. Sagoe-Moses C, Pearson RD, Perry J, Jagger J. Risks to Health Care Workers in Developing Countries. N Engl J Med. 200; 345(7):538-41.

11. Minehata M, Sture J, Shinomiya N, Whitby S. Implementing Biosecurity Education: Approaches, Resources and Programmes. Sci Eng Ethics. 2013; 19(4):1473-86.

12. Hardefeldt L, Nielsen T, Crabb H, Gilkerson J, Squires R, Heller J, et al. Veterinary students' knowledge and perceptions about antimicrobial stewardship and biosecurity-a national survey. Antibiotics (Basel). 2018; 7(2): pii: E34

13. Díaz JM, Vásquez MEP, Lea P, Muñoz Z, Emperatriz J. Conocimiento y actitudes hacia accidentes ocupacionales biológicos en estudiantes de enfermería de la Universidad Nacional de la Amazonía Peruana enfermeras internas, entre quienes se mería está especialmente expuesto y es el declaraciones de accidentes. Conoc Amaz. 2012; 3(2):169-80.

14. Modi PD, Kumar P, Solanki R, Modi J, Chandramani S, Gill N. Hand Hygiene Practices Among Indian Medical Undergraduates: A Questionnaire-Based Survey. Cureus. 2017; 9(7): e1463.

15. Chero Pacheco VH. Nivel de conocimiento sobre medidas de bioseguridad y su empleo por parte de los estudiantes en una Universidad Peruana. Ágora Rev Científica. 2017; 3(2):361

16. Goyal S, Khot SC, Ramachandran V, Shah KP, Musher DM. Bacterial contamination of medical providers' white coats and surgical scrubs: A systematic review. Am J Infect Control. 2019; 47(8):994-1001.

17. Olvera-Lopez E, McCaffery E, Kasubhai M. White coat wearing and laundering frequency among internal medicine department physicians. Am J Infect Control. 2019 Nov. pii: S0196-6553(19)30927-7.

18. Treakle AM, Thom KA, Furuno JP, Strauss SM, Harris AD, Perencevich EN. Bacterial contamination of health care workers' white coats. Am J Infect Control. 2009 1; 37(2):101-5.

Recibido: 13/02/2020

Aceptado: 20/02/2020 